COMUNICACIÓN CIENTÍFICA IX CONGRESO NACIONAL DE LA SOCIEDAD CIENTÍFICA ESPAÑOLA DE ENFERMERÍA - SCELE. Mayo de 2018. UNIVERSIDAD DE ALICANTE.

\title{
ELABORACIÓN DE UN PROTOCOLO DE FOTOTERAPIA NEONATAL
}

Ma José Castaño Picó

M. Sánchez Maciá

Hospital General Universitario de Elche 


\section{PALABRAS CLAVE:}

Protocolo; ictericia neonatal; cuidados de enfermería

Objetivos: Identificar la evidencia científica en el empleo de fototerapia en el tratamiento de la ictericia neonatal para elaborar un protocolo de Enfermería.

Métodos: Revisión bibliográfica durante el período 2011-2016, sin límites en el idioma, en las bases de datos PubMed con las palabras clave "neonatal jaundice guidelines", "neonatal jaundice phototherapy"; Cuiden, con las palabras clave "neonatal jaundice", "neonatal phototherapy"; y en Google Académico con "protocolo" "ictericia neonatal", "guidelines neonatal jaundice", "guidelines phototherapy".

Resultados: Se seleccionaron 3 directrices italianas, noruegas y de los Países Bajos; 7 guías de práctica clínica de Estados Unidos, Canadá, Reino Unido, Irlanda, Australia, y Malasia; 2 vías clínicas de Estados Unidos y el Reino Unido; 2 protocolos de enfermería de España; y10 revisiones para su estudio mediante lectura crítica.

Se elabora un protocolo de enfermería para fototerapia en recién nacidos mayores de 35 semanas de edad gestacional, fundamentado en la evidencia científica disponible en la revisión de la literatura, y consensuado por todo el personal de enfermería de la Unidad, donde no se han desarrollado protocolos contextualizados de actuación de enfermería para la aplicación de fototerapia.

Discusión: La ictericia neonatal es frecuente en la etapa perinatal, y supone un considerable factor de riesgo para la encefalopatía aguda por bilirrubina, pudiendo transformarse en crónica (kernicterus), que ocasiona un sustancial riesgo en la mortalidad de los recién nacidos.

Se produce controversia respecto la distancia óptima entre la lámpara de fototerapia y el recién nacido, y la frecuencia del cuidado ocular y del control de la temperatura.

No existe un procedimiento estándar para la aplicación de fototerapia, pero existe evidencia de que es esencial aumentar, tanto el área cutánea expuesta como la energía proporcionada, para mejorar la efectividad de la fototerapia. Por lo tanto, los cuidados abarcados en el protocolo se aplican con ésta finalidad, concordando con los protocolos de Albacete y Ciudad Real, la vía clínica del Reino Unido, las guías de práctica clínica de Estados Unidos, Canadá, Reino Unido,

Australia, y Malasia, y con las revisiones de Ali, Bhutani, Duran, Maisels, Muchowski, Stokowski, y Ullah.

\section{Conclusiones:}

El cuidado de enfermería es esencial tanto para el mantenimiento y el éxito del proceso de fototerapia, como para disminuir las complicaciones. Un protocolo consensuado puede mejorar los resultados de fototerapia y disminuir la variabilidad de los cuidados, mejorando la calidad de la atención sanitaria, y por tanto, el costeefectividad de los servicios de salud. 